\title{
Análise do perfil físico e cardiovascular de policiais militares na formação BOPE*
}

\section{Analysis of physical profile and cardiovascular of police military training in BOPE}

Frederico Santiago ${ }^{1}$

Mateus Medeiros Leite ${ }^{2}$

Vinícius Borges Vieira ${ }^{3}$

Alessandro de Oliveira Silva ${ }^{4}$ Tácio Rodrigues da Silva Santos ${ }^{5}$

Darlan Lopes de Farias ${ }^{6}$
Recebido em: 26/09/2016.

Aprovado em: 21/11/2016.

1 Aluno do curso de Educação Física do Centro Universitário de Brasília - UniCEUB, Brasil, Brasília- DF.

2 Aluno do curso de Educação Física do Centro Universitário de Brasília - UniCEUB, Brasil, Brasília- DF.

3 Aluno do curso de Educação Física do Centro Universitário de Brasília - UniCEUB, Brasil, Brasília- DF.

4 Professor do Centro Universitário de Brasília UniCEUB, Brasil, Brasília- DF.

5 Professor do Centro Universitário de Brasília UniCEUB, Brasil, Brasília- DF.

6 Professor do Centro Universitário de Brasília UniCEUB, Brasil, Brasília- DF; email: fariasdl@ gmail.com.

\section{Resumo}

Os índices de obesidade vêm aumentando, de forma alarmante, na maioria dos países e vários estudos têm encontrado, em suas análises, índices preocupantes de aptidão e composição física de militares. Por conseguinte, o presente estudo teve como objetivo verificar e associar as variáveis de perfil físico e cardiovascular de policiais militares ingressantes voluntários em curso do BOPE do Distrito Federal. A amostra foi composta por 25 (vinte e cinco) policias militares ingressantes e foram coletadas a força de preensão manual (JAMAR ${ }^{\circ}$ ), circunferências (Sanny ${ }^{\circ}$ ) e pressão arterial em repouso (Microlife ${ }^{\odot}$ ). Os resultados apontam que os policiais apresentam níveis de pressão arterial e força muscular desejáveis, mas composição corporal comprometida, o que pode refletir na execução dificultada da atividade profissional.

Palavras-chave: Polícia. Força Muscular. Composição corporal. Saúde Pública.

\begin{abstract}
Obesity rates are increasing alarmingly in most countries and several studies have found analyzes worrying levels of fitness and military physical composition. Therefore, the present study was to evaluate and associate as physical profile variables and cardiovascular Military Police freshmen volunteers in progress to BOPE Federal District. One was composed of 25 ( Twenty Five ) entering military police and Were collected Sample hold Manual Force ( Jamar $^{\circledR}$ ), circumferences ( Sanny ${ }^{\oplus}$ ) in blood pressure and Home (Microlife ${ }^{\oplus}$ ). The results show that the police have levels of blood pressure and strength force normal, but committed Body composition which may reflect implementation hampered the professional activity.
\end{abstract}

Keywords: Police. Muscle strength. Body composition. Public health. 


\section{Introdução}

Os índices de obesidade vêm aumentando, de forma alarmante, na maioria dos países, alcançando mais de um bilhão de pessoas em todo o mundo (WHO, 2015). Segundo dados da Pesquisa Nacional de Saúde (PNS) realizada em 2013 e anunciados pelo IBGE em 2016, quase $60 \%$ da população brasileira está nos níveis de sobrepeso ou obesidade (IBGE, 2013).

Essas estatísticas permitem que sejam desenvolvidos estudos em diversas populações objetivando a mudança desses níveis. Em militares foram realizados vários estudos e verificou-se que alguns movimentos físicos são primordiais para o bom exercício da profissão (saltar, puxar, empurrar, correr, arrastar, elevar) e estes são prejudicados se o policial não mantém um estilo de vida ativo. Vários estudos têm encontrado, em suas análises, índices preocupantes de aptidão e composição física de militares (DAWES, 2016; ZOREC, 2009; SANTOS, 2013).

Tahan (2015) relatou que os policiais são trabalhadores que expõem, demasiadamente, o adoecimento físico e mental, e, ainda, informa que características inerentes a essa profissão podem desencadear agravos à saúde. Neste estudo ele avaliou 120 militares por meio de indicadores antropométricos (IMC, CC, RCQ) e encontrou alta prevalência de risco cardiovascular.

Recentemente, Dawes (2016), em um elegante estudo, detectou que o aumento do percentual de gordura estava relacionado com a diminuição do desempenho em diversos testes de aptidão física. Foram analisados dados de testes físicos e medidas corporais de 76 policiais da cidade do Colorado (EUA).

Santos (2013) analisaram 51 policiais militares de Boa Vista (Roraima - Brazil) e, apesar de apresentarem bons resultados de aptidão física, a maioria apresentou excesso de peso. Argumentaram que, talvez, a grande quantidade de massa magra possa ter influenciado em bons resultados nos testes físico, mas, infelizmente, o referido estudo tem a limitação de não ter avaliado o percentual de gordura e, apenas, o IMC (Índice de Massa Corporal) na presente análise, não é o adequado.

Um estudo mais antigo de Zorec (2009) objetivou analisar as medidas de composição corporal de militares ( $\mathrm{n}=172$ de 20 a 50 anos) e operadores de máquinas ( $\mathrm{n}=91$ de 20 a 50 anos) em diferentes fórmulas e encontrou resultados surpreendentes mostrando que os militares precisam de um melhor treinamento para que se enquadrem nas medidas visadas para a saúde. Ademais, os operadores apresentaram valores dentro dos padrões normais.

O batalhão de operações especiais é um destacamento de elite da polícia militar e é reconhecido mundialmente pelo alto nível de responsabilidade em nível de comando. Após o curso, o policial será capaz de executar e planejar missões especiais que venham exigir um elevado preparo técnico, tático e psicológico, tendo em vista a atuação dirigida para as ações no campo da segurança pública (SANTOS, 2007).

Nesse sentido, para ser convocado a participar do curso, o aluno deve passar por uma bateria de exames médicos e físicos. Após essa fase, o policial entra no curso de formação de dura em média de 3 a 6 meses de curso.

Ademais, o objetivo do presente estudo é analisar qual o perfil do policial que ingressa no curso de formação do batalhão de operações especiais e verificar se a tendência dos estudos supracitados prevalece nessa região, além de tecer possíveis planejamentos para melhora e/ou manutenção dos resultados encontrados.

\section{Metodologia}

Esta foi uma pesquisa quantitativa observacional de corte prospectivo, autorizada pelo comitê de ética do UniCEUB sob o número 077578/2015 realizada com 25 militares $(31,20 \pm 5,68$ anos) do Distrito Federal. Foram avaliados em composição corporal, força muscular relativa, frequência cardíaca e pressão arterial de repouso.

\subsection{Composição corporal}

A medida da massa corporal foi realizada com o voluntário descalço, vestindo roupas leves, e utilizando-se balança digital (Welmy-W110H, São Paulo, Brasil) com capacidade de $150 \mathrm{~kg}$ e divisão de $100 \mathrm{~g}$. A estatura foi medida por um estadiômetro de parede (Sanny, São Paulo, Brasil), com capacidade de $2200 \mathrm{~mm}$ e divisão de $1 \mathrm{~mm}$. Com essas medidas, foram realizados os cálculos para identificação do Índice de Massa Corporal (IMC) utilizando a fórmula $\left[\mathrm{Kg} /(\text { estatura })^{2}\right]$. As circunferências e as dobras cutâneas foram medidas com o voluntário na posição ereta, com o mínimo de roupa possível, seguindo o protocolo de Pollock, (1978), para determinação do percentual de gordura e densidade corporal. 


\subsection{Força de Preensão Manual}

A força de preensão manual foi obtida com dinamômetro hidráulico manual (Jamar), respeitando o protocolo de España-Romero e colaboradores (2009). Para tanto, os voluntários permaneceram sentados e com o cotovelo em flexão de 45 graus. Para todos os participantes, a empunhadura do dinamômetro foi ajustada, individualmente, de acordo com o tamanho das mãos de forma que a haste mais próxima do corpo do dinamômetro ficasse posicionada sobre as segundas falanges dos dedos indicador, médio e anular. O período de recuperação entre as medidas foi de 30 a 60 segundos. O teste foi realizado em três tentativas na mão que o participante considerou mais forte. A média entre as três tentativas foi utilizada como medida. A força relativa (FR) foi calculada com a equação $\mathrm{FR}=$ [força muscular absoluta (kg)/massa corporal (kg)]. A coleta dos dados da força de preensão manual foi realizada por um avaliador experiente e treinado (PRESTES; TIBANA, 2013).

\subsection{Medidas cardiovasculares}

A determinação da pressão arterial sistólica (PAS) e diastólica (PAD) foi realizada pelo método oscilométrico, adotando-se a metodologia proposta pela VI Diretriz Brasileira de Hipertensão Arterial (SBC, 2010) e um medidor oscilométrico (Microlife 3AC1-1, Widnau, Suiça), validado pela European Society of Hypertension. Com o voluntário na posição sentada, após 10 minutos de repouso, braço direito apoiado e ao nível do coração, braçadeiras apropriadas ao tamanho do braço, colocar-se-á a braçadeira do aparelho a cerca de $3 \mathrm{~cm}$ acima da fossa antecubital centralizando a bolsa de borracha sobre a artéria umeral. Os valores das medidas de PAS e PAD serão utilizados para o cálculo da pressão arterial média (PAM) pela equação: $\mathrm{PAM}=\mathrm{PAD}+[(\mathrm{PAS}-\mathrm{PAD}) \div 3]$. A frequência cardíaca de repouso foi medida, também, pelo mesmo aparelho.

\subsection{Análise Estatística}

Inicialmente, os dados foram tratados a partir dos procedimentos descritivos, com as informações sendo processadas no pacote computacional BioEstat em sua versão 5.3. Medidas de tendência central e variabilidade dos dados foram representadas como média e desvio padrão. Foi utilizado coeficiente de correlação de Spearman para correlação entre as variáveis. $\mathrm{O}$ nível de significância adotado foi de $(\mathrm{P} \leq 0,05)$.

\section{Resultados}

Os dados da pesquisa, para as características antropométricas, obtidas por meio da mensuração de peso, estatura, dobras cutâneas e circunferências dos participantes estão expostos na Tabela 1, apresentados com médias e desvio padrão.

Tabela 1. Características antropométricas.

\begin{tabular}{l|c}
\hline Variáveis & Valores \\
\hline Massa (Kg) & $79,31 \pm 8,18$ \\
\hline Estatura (m) & $1,77 \pm 0,05$ \\
\hline IMC (kg/m²) & $25,19 \pm 1,96$ \\
\hline RCQ & $0,89 \pm 0,03$ \\
\hline CP (cm) & $39,63 \pm 2,05$ \\
\hline DC & $1,05 \pm 0,01$ \\
\hline \% Gordura & $20,71 \pm 5,12$ \\
\hline
\end{tabular}

Para cálculo da força muscular relativa, foi utilizada a equação $\mathrm{FR}=$ [força muscular absoluta $(\mathrm{kg}) /$ massa corporal $(\mathrm{kg})]$ e a força absoluta foi medida por meio do teste de força de preensão manual na mão que o participante considerava a mais forte. Os valores obtidos estão demonstrados na Tabela 2 apresentados com médias e desvio padrão, assim como os valores das medidas cardiovasculares dos participantes, no que diz respeito à pressão arterial e frequência cardíaca.

Tabela 2. Valores de Medidas de força e cardiovasculares.

\begin{tabular}{l|c}
\hline Variáveis & Valores \\
\hline FA (Kg) & $91,71 \pm 15,67$ \\
\hline FR & $1,16 \pm 0,19$ \\
\hline PAS & $12,12+1,27$ \\
\hline PAD & $7,00+0,87$ \\
\hline PAM & $8,71+0,93$ \\
\hline FC & $67,56+11,87$ \\
\hline
\end{tabular}

Legenda: $F A=$ Força absoluta; $F R=$ Força relativa; $P A S=$ Pressão arterial sistólica; $\mathrm{PAD}=$ Pressão arterial diastólica; $\mathrm{PAM}=$ Pressão arterial média; FC= Frequência cardíaca.

No que diz respeito à correlação entre força relativa com outras variáveis, não foi demonstrada correlação, estatisticamente significativa, entre FR e \% gordura (rs = 
$-0,3522 ; \mathrm{p}=0,0841)$, FR e RCQ ( $\mathrm{rs}=-0,3337 ; \mathrm{p}=0,1030)$, FR e CP (rs = - 0,3891; $\mathrm{p}=0,0545)$, porém houve correlação negativa fraca entre FR e PAM ( $\mathrm{rs}=-0,4769 ; \mathrm{p}=$ 0,0159), FR e PAS ( $r s=-0,4455 ; \mathrm{p}=0,0256)$, FR e PAD ( $\mathrm{rs}=-0,4442 ; \mathrm{p}=0,0260)$.

Correlacionando as medidas cardiovasculares com as variáveis antropométricas, não foi apresentado correlação, estatisticamente significativa, entre FC e CP ( $\mathrm{rs}=0,0501 ; \mathrm{p}=0,8120), \mathrm{FC}$ e RCQ ( $\mathrm{rs}=0,3601 ; \mathrm{p}=$ 0,0769), FC e \% de Gordura ( $r s=0,1987 ; \mathrm{p}=0,3410$ ). Entre PAM e CP, não foi demonstrada correlação (rs = $0,2549 \mathrm{p}=0,2187$ ) assim como PAM e RCQ ( $r s=0,3153$; $\mathrm{p}=0,1247)$ e PAM e $\%$ de Gordura ( $\mathrm{rs}=0,3821 ; \mathrm{p}=$ 0,0593).

\section{Discussão}

O objetivo do presente estudo foi analisar o perfil físico e cardiovascular de policiais militares em curso de formação do batalhão de operações especiais e verificar se os resultados seguem a tendência da maioria dos estudos. O perfil físico apresentou resultados que tendem a ser elevados de IMC, CP, RCQ e \% Gordura seguindo a tendência geral dos estudos com militares. As medidas cardiovasculares apresentaram uma média nos valores normais.

A composição corporal determina o comportamento das diversas formas do movimento humano. Muitos autores citam que uma boa composição corporal associada a um bom condicionamento físico é capaz de reduzir risco de desenvolver doenças relacionadas a atividade profissional, além do aspecto biopsicossocial (PESCATELLO, 2004; TAHAN, 2015). No entanto, os resultados do presente estudo refletem uma tendência preocupante da composição corporal visto que estes irão ingressaram em um curso de operações especiais e a valência prontidão, possivelmente, apresentar-se-á comprometida.

A circunferência do pescoço é uma medida simples que possibilita a identificação de sobrepeso e obesidade além de estar correlacionada com risco cardiovascular, indicador do consumo alimentar desregrado, predição de resistência à insulina e marcador de síndrome metabólica (BAENA, 2016; DAWES, 2016). Ben Noun e Laor (2006), em seu estudo longitudinal, em 364 participantes entre homens e mulheres, encontrou valores de circunferência do pescoço de $39 \mathrm{~cm}$ e, após análises, verificou, também, que a circunferência do pescoço foi positivamente correlacionada com fatores de risco cardiovascular e síndrome metabólica. No presente estudo, apesar de não apresentar correlação, o valor coincide com o de Ben Noun e Laor (2006), permitindo inferir que estão no padrão limite, pois os valores acima de 39 indicam alto riso cardiovascular.

Um novo questionamento dessa medida foi, recentemente, publicado por Baena (2016) informando que a circunferência do pescoço não é correlacionada com fatores de risco cardiovascular. Estudo bem significativo e bem conduzido com 8726 indivíduos saudáveis. Alertam que, apesar de a medida ser simples e prática, é necessário observar com cautela essa medida, pois ela apresenta limitações.

Nas medidas de gordura corporal, foi encontrado um valor $(20,71 \%)$ preocupante, pois em homens saudáveis e em alguns estudos com a mesma população, apresentam valores de gordura entre 15 e 17\%. Essas medidas podem influenciar na qualidade de vida geral do policial.

No entanto, as medidas cardiovasculares apresentaram dentro dos padrões normais para adultos saudáveis. Logo, apesar de a composição corporal apresentar níveis altos e não indicados para a população em geral, faZ-se necessário um acompanhamento mais robusto dessa população com o intuito de melhor entendimento desse comportamento.

Outrossim a força muscular tem despertado pesquisas relacionando-a com a saúde (PRESTES; TIBANA, 2013), o que não ocorreu com as correlações realizadas, apesar de haver uma tendência fraca negativa demonstrando que quanto maior a força muscular menor será os níveis pressóricos.

Torna-se interessante ressaltar há a necessidade no período de formação do policial, que sejam submetidos a esforços físicos próximos a intensidades máximas, pois o cotidiano exige essas capacidades. Todavia, qualquer atividade máxima deve ser planejada, sistematizada e monitorada, a fim de que situações fortuitas não ocorram. Logo, uma análise preliminar não só no âmbito social e/ou burocrático não é suficiente para que o policial ingresse em um curso de operações especiais, mas sim uma minuciosa do perfil físico constantemente.

Outro fator importante refere-se ao fato de que, apesar de existirem algumas pesquisas com militares, ain- 
da é escasso este estudo. Há uma necessidade emergente de pesquisas que levem em consideração as diversas corporações, regiões e culturas o que torna quase inviável a realização de um estudo desse porte.

Enfim, foram encontradas fortes limitações no presente estudo. O tamanho da amostra, o perfil da amostra transversal, não permitindo análise de causa e efeito e a ausência de análises invasivas como análises sanguíneas e/ou de imagem corporal.

\section{Conclusão}

O presente estudo apresentou perfil físico comprometido e perfil cardiovascular normal. Logo, parece relevante sugerir novas análises dessa população para que os perfis físico e cardiovascular possam estar em padrões saudáveis para essa população.

Medidas de força e sua relação com medidas cardiovasculares são avaliações populacionais que deveriam fazer do "rol" de testes militares, no entanto, nessa população são essenciais, visto que direcionam o treinamento assim como determinam as capacidades e limites de cada policial. Ademais, os resultados apresentados demonstraram uma discrepância dessas relações que serve de alerta a esse grupo de profissionais.

\section{Referências}

BAENA, C. P. et al. Neck circumference is associated with carotid intimal-media thickness but not with coronary artery calcium: Results from The ELSA-Brasil. Nutrition, Metabolism \& Cardiovascular Diseases, Amsterdam, v. 26, n. 3, p. 216-222, Mar. 2016. doi: 10.1016/j.numecd.2016.01.004

BEN-NUON, L.; LAOR, A. Relationship between changes in neck circumference and cardiovascular risk factors. Experimental \& Clinical Cardiology, Bern, v. 11, n. 1, p. 14-20, Jan. 2006.

DAWES J. J. et al. Associations between anthropometric characteristics and physical performance in male law enforcement officers: a retrospective cohort study. Annals of Occupational and Environmental Medicine, London, v. 28, n. 26, p. 1-7, Jun. 2016. doi: 10.1186/s40557016-0112-5

ESPAÑA-ROMERO, V. et al. Elbow position affects handgrip strength in adolescents: validity and reliability of Jamar, DynEx, and TKK dynamometers. The Journal of Strength \& Conditioning Research, v. 24, n. 1, p. 272277, Dec. 2009. doi: 10.1519/JSC.0b013e3181b296a5
FRIZON, V.; BOSCAINE, C. Circunferência do Pescoço, Fatores de Risco para Doenças Cardiovasculares e Consumo Alimentar. Revista Brasileira de Cardiologia, Rio de Janeiro, v. 26, n. 6, p. 426-434, nov./dez. 2013

INSTITUTO BRASILEIRO DE GEOGRAFIA E ESTATÍSTICA. Pesquisa Nacional de Saúde 2013. Disponível em: <http://biblioteca.ibge.gov.br/biblioteca-catalogo .view $=$ detalhes\&id $=291110>$. Acesso em: 07 set. 2016.

MION JR., D. et al. V Diretrizes brasileiras de hipertensão arterial. Arquivos Brasileiros de Cardiologia, São Paulo, v. 89, n. 3, p. e24-e79, set. 2007. doi: 10.1590/ S0066-782X2007001500012

PEREIRA, D. C. R. Circunferência do pescoço como possível marcador para síndrome metabólica em universitários. Revista Latino-Americana de Enfermagem, Ribeirão Preto, v. 22, n. 6, p 973-979, nov./dez. 2014. doi: 10.1590/0104-1169.3565.2505

PESCATELLO, L. S. et al. American College of Sports Medicine position stand. Exercise and hypertension. Medicine and Science in Sports and Exercise, Madison, v. 36, n. 3, p. 533-553, Mar. 2004. doi: 10.1249/01. MSS.0000115224.88514.3A

POLLOCK, M. L.; JACKSON, A. S. Generalized equations for predicting body density of men. The Bristish Journal of Nutrition, Wallingford, v. 40, n. 3, p. 497-504, Nov. 1978. doi: 10.1079/BJN19780152

PRESTES, J.; TIBANA, R. A. Muscular static strength test performance and health: absolute or relative values? Revista da Associação Médica Brasileira, São Paulo, v. 59, n. 4, p. 308-309, jul./ago. 2013. doi: 10.1016/j. ramb.2013.01.009

SANTOS, J. A. B. et al. Nível de adiposidade corporal e de aptidão física de policiais mlitares de Boa Vista - Roraima. Revista Brasileira de Prescrição e Fisiologia do Exercício, São Paulo, v. 7, n. 37, p. 21-28, jan./fev. 2013.

SANTOS, M. R.; FILHO J. F. Estudo do perfil dematoglífico, somatotípico e das qualidades físicas dos policiais do batalhão de operações especiais (PMERJ) no ano de 2005. Fitness \& Performance Journal, Rio de Janeiro, v. 6, n. 2, p. 98-104, mar./abr. 2007. doi:10.3900/fpj.6.2.98.p

SILVA, C. C. et. al. Circunferência do pescoço como um novo indicador antropométrico para predição de resistência à insulina e componentes da síndrome metabólica em adolescentes: Brazilian Metabolic Syndrome Study. Revista Paulista de Pediatria, São Paulo, v. 32, n. 2, p. 221229, jun. 2014. doi: 10.1590/0103-0582201432210713 
SOCIEDADE BRASILEIRA DE CARDIOLOGIA. VI Diretriz Brasileira de Hipertensão Arterial, Arquivos Brasileiros de Cardiologia, São Paulo, v. 95, n. 1, Supl. 1, p. 1-51, dez. 2010. doi:10.1590/S0066-782X2010001700001

TAHAN, F.; PEREIRA, J. C. Avaliação do risco cardiovascular por indicadores antropométricos em policiais militares de um batalhão do Sul de Minas. Nutrição Brasil, São Paulo, v. 14, n. 4, p. 230-236, out./dez. 2015
WORLD HEALTH ORGANIZATION. Obesity and overweight. Disponível em: <http://www.who.int/mediacentre/factsheets/fs311/en/>. Acesso em: 07 set. 2016.

ZOREC, B. Anthropometric characteristics in police officers. Journal of Criminal Justice and Security, Ljubljana, v. 1, p. 26-35, mar. 2009. 